\title{
Global, regional, and national consumption of controlled opioids: a cross-sectional study of 214 countries and non-metropolitan territories
}

British Journal of Pain

1-7

(c) The British Pain Society 2021

\section{(c) (i)}

Article reuse guidelines:

sagepub.com/journals-permissions DOI: $10.1177 / 20494637211013052$ journals.sagepub.com/home/bjp

(S)AGE

\author{
Georgia C Richards ${ }^{1,2}$ (D), Jeffrey K Aronson ${ }^{2}$, \\ Kamal R Mahtani' ${ }^{2}$ and Carl Heneghan ${ }^{1,2}$
}

\begin{abstract}
Introduction: The consumption of opioids has increased globally since the 1990s. Previous studies of global opioid consumption have concentrated on morphine alone or a subset of opioids, with a focus on cancer pain and palliative care. In this study, we have determined the global, regional, and national consumption of all controlled opioids, including anaesthetics, analgesics, antidiarrheals, opioid substitution therapies, and cough suppressants.

Methods: We conducted a cross-sectional study using data from the International Narcotics Control Board (INCB). We calculated mean opioid consumption (mg/person) globally, regionally, and nationally for 2015-2017, where consumption refers to the total amount of controlled opioids distributed for medical purposes and excludes recreational use. We ranked countries by total consumption and quantified the types of opioids consumed globally.

Results: Between 2015 and 2017, 90\% of the world's population consumed only $11 \%$ of controlled opioids. An average of $32 \mathrm{mg} /$ person was consumed annually, but this was not equally distributed across the world. Consumption was the highest in Germany ( $480 \mathrm{mg} /$ person), followed by Iceland ( $428 \mathrm{mg} /$ person), the United States (398 mg/person) and Canada (333 mg/person). Oxycodone (35\%) was the most heavily consumed controlled opioid globally, followed by morphine $(15.9 \%)$, methadone $(15.8 \%)$ and tilidine (14\%).

Conclusion: Large disparities persist in most of the world in accessing essential opioid medicines. Consumption patterns should continue to be monitored, and collaborative strategies should be developed to promote access and the appropriate prescribing of opioids in all countries and non-metropolitan territories.
\end{abstract}

\section{Keywords}

Opioids, pain management, anaesthetics, analgesics, antidiarrheals, opioid substitution therapies, cough suppressants, narcotics

\section{Introduction}

Access to medicines is widely recognized as a human right, ${ }^{1}$ although many countries experience barriers to access. The World Health Organization (WHO) considers five opioids to be essential medicines, including codeine, fentanyl, loperamide, methadone, and morphine, which are listed in its Model List of Essential Medicines. ${ }^{2,3}$ The WHO's analgesic ladder for cancer
1 Global Centre on Healthcare and Urbanisation, Kellogg College, University of Oxford, Oxford, UK

${ }^{2}$ Centre for Evidence-Based Medicine, Nuffield Department of Primary Care Health Sciences, University of Oxford, Oxford, UK

Corresponding author:

Georgia C Richards, Global Centre on Healthcare and Urbanisation, Kellogg College, University of Oxford, 60-62 Banbury Road, Oxford OX2 6PN, UK.

Email: georgia.richardsakellogg.ox.ac.uk 
pain and the recognition that pain relief is a patient and human right led to a global increase in opioid consumption during the $1990 \mathrm{~s},{ }^{4-6}$ particularly in the Americas, Europe and Oceania, which account for most of the consumption. ${ }^{7-10}$ But this growth in opioid consumption was less pronounced in most of Asia and Africa.

Since 1961, most opioids have been internationally controlled under the United Nations (UN) Single Convention on Narcotic Drugs, amended by the 1972 Protocol. ${ }^{11}$ Countries and territories that sign the treaty commit to implementing the drug control measures while ensuring access for medical and scientific purposes. The International Narcotics Control Board (INCB), an independent body of the UN, is responsible for monitoring implementation and compliance with international drug control treaties, which requires governments to report annual statistics on narcotic consumption relating to controlled drugs. ${ }^{12}$ When using data from the INCB, the term 'consumption' is used as per article 1, paragraph 2 of the Single Convention and 1972 Protocol; a drug is considered as 'consumed' when it has been supplied to any person or enterprise for retail distribution, medical use or scientific research. ${ }^{13}$ To date, most pharmacoepidemiological studies on opioid use have been conducted in high-income countries owing to data provisions. ${ }^{14-20}$ Therefore, the use of INCB data allows opioid consumption to be compared globally, regionally and nationally for countries in all income groups.

Several observational studies using data from the INCB have evaluated barriers to access and have shown complex interactions between historical, social, cultural, economic and political decisions, limiting medical access to opioids. ${ }^{8,9,21-31}$ However, most studies on controlled opioid consumption have focused on cancer pain and palliative care and have examined morphine alone or a subset of five opioids, ${ }^{9,10,21,32}$ which may underestimate the scale of the problem. To the best of our knowledge, no studies have evaluated controlled opioid consumption trends that simultaneously include opioids for anaesthesia, analgesia, the management of diarrhoea, opioid dependence and cough suppression. Therefore, this study aimed to determine global, regional and national consumption of all controlled opioids, where consumption refers to the total amount of opioids distributed for medical purposes and excludes recreational use.

\section{Methods}

\section{Study design and data sources}

We designed and conducted a cross-sectional observational study using data from the INCB. ${ }^{33}$ The INCB is an independent body of the UN, and it monitors the implementation of international drug control conventions, including the Single Convention on Narcotic Drugs of 1961, which requires governments to report annual statistics on narcotic consumption relating to controlled drugs. ${ }^{12}$ Consumption refers to the total amount of a narcotic distributed for medical purposes at the retail level (i.e. to institutions and programmes that are licensed to dispense to patients). ${ }^{13}$ We obtained data on consumption in kilograms from 2015 to 2017 and removed 26 non-opioid substances (e.g. cannabis, coca leaf and cocaine) to create a data set of all opioids consumed. The included and excluded substances are listed in Box S1 in the Supplement.

\section{Data analysis}

We included all countries and non-metropolitan territories $(n=214)$ that provided data to the INCB. We converted raw consumption data from $\mathrm{kg}$ to $\mathrm{mg}$ and divided by global, regional and country-specific populations for 2016 to calculate mean rates of consumption (mg/person). The INCB recommends using a 3-year mean to display the data, and previous studies have used this to account for annual variations in reporting, providing more stable data. ${ }^{23}$ We determined the medians and interquartile ranges (IQR) for the consumption of controlled opioids worldwide. We categorized countries into deciles based on their consumption using the xtile function in Stata ${ }^{34}$ and calculated the percentage of opioids consumed and the percent of the population in each decile. We ranked countries by the rate of consumption (mg/person). We categorized opioids into their Anatomical Therapeutic Classification (ATC) index subgroups (i.e. analgesics, opioid substitution therapies, cough suppressants, anaesthetics and antidiarrheals) and ranked types of opioids by volume of consumption.

\section{Software and data sharing}

We used Stata $v 16^{35}$ for all statistical analyses and pandas and plotly modules in Jupyter Notebooks with Python v3 for choropleth maps. The study materials, data and statistical code are all openly available on the Open Science Framework $^{36}$ and GitHub. ${ }^{37}$ We used the STROBE reporting guidelines in writing our manuscript (see the Supplement for the completed checklist).

\section{Results}

Globally, over 700 tonnes $(710,043 \mathrm{~kg})$ of controlled opioids were consumed in 2015-2017, an average of $32 \mathrm{mg} /$ person each year. Regionally, the Americas had the greatest average consumption (144 $\mathrm{mg} /$ person), followed by Oceania (132 mg/person), Europe ( $98 \mathrm{mg} /$ person), Asia ( $3.5 \mathrm{mg} /$ person), and Africa $(1.4 \mathrm{mg} /$ person) (see Figure $S 1$ in the Supplement). 


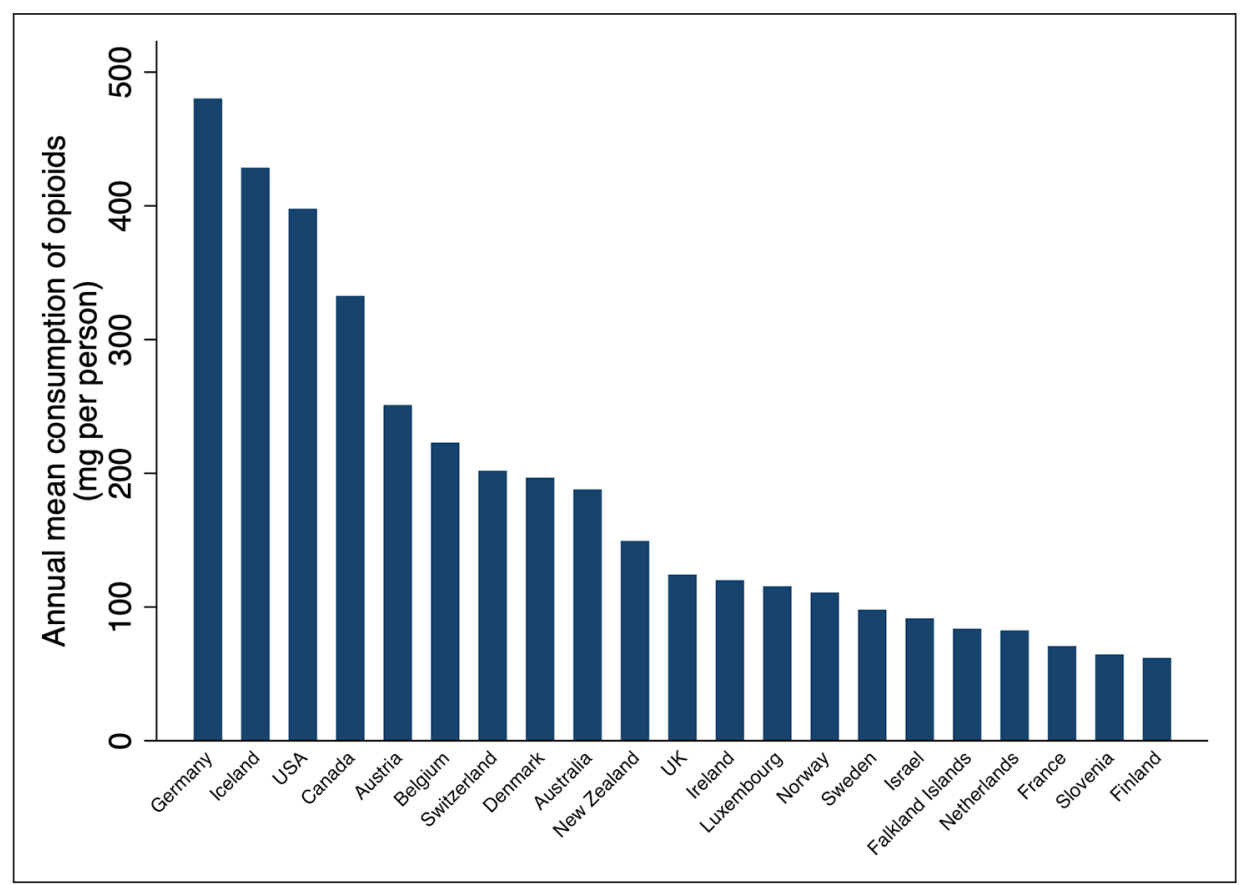

Figure 1. Countries ( $n=21$ ) in the top decile for controlled opioid consumption (mg per person) for 2015-2017. Countries in the top decile consumed $89 \%$ of controlled opioids between 2015 and 2017 and accounted for only $10 \%$ of the 2016 world population. Consumption refers to the total amount of controlled opioids distributed for medical purposes and excludes recreational use; it was calculated by determining the 3-year mean for 2015-2017 and dividing this by the 2016 population for each country. Data were obtained from the International Narcotics Control Board.

Nationally, countries consumed a median of 3.3 $\mathrm{mg} /$ person $(\mathrm{IQR}=0.24-14.8$; range $=0-480 \mathrm{mg} / \mathrm{per}-$ son). Consumption of controlled opioids was the highest in Germany ( $480 \mathrm{mg} /$ person), followed by Iceland (428 mg/person), the United States (398 mg/ person), and Canada (333 mg/person) (see Figure 1 and Table $S 1$ in the Supplement). There were 21 countries in the top decile of consumption; they consumed $89 \%$ (630 tonnes) of the world's controlled opioids and accounted for only $9.7 \%$ of the world's population ( 687 million of 7.1 billion people in 2016) (see Table S2 in the Supplement). Deciles that accounted for larger percentages of the population, decile 6 ( $24 \%$ of the population), decile 3 ( $21 \%$ of the population), and decile 4 (13\% of the population), consumed only $2.7 \%, 0.28 \%$, and $0.32 \%$ of the world's controlled opioids, respectively (see Table S2 in the Supplement). Thirty-five countries, accounting for $3.1 \%$ of the population, reported no consumption of controlled opioids to the INCB. Figure 2 displays the distribution of controlled opioids consumed for all countries and territories by decile.

Globally, oxycodone was the most heavily consumed, accounting for one-third of all opioids, followed by morphine $(15.9 \%)$, methadone $(15.8 \%)$, and tilidine $(13.9 \%)$ (see Figure 3 and Table S3 in the Supplement). Analgesics (493 tonnes, $\mathrm{n}=12$ opioids) were the most common Anatomical Therapeutic Chemical (ATC) category of opioids consumed, followed by opioid substitution therapies (114 tonnes, $\mathrm{n}=2$ opioids), cough suppressants (95 tonnes, $\mathrm{n}=2$ opioids), anaesthetics (0.3 tonnes, $\mathrm{n}=3$ opioids), and antidiarrheals ( 0.07 tonnes, $\mathrm{n}=2$ opioids) (see Table $\mathrm{S} 3$ in the Supplement).

\section{Discussion}

The global consumption of opioids was disproportionately distributed; 6.4 billion people worldwide had little or no access to controlled opioids for medical uses. Yet an average of $32 \mathrm{mg}$ for every person in 2016 was available. Consumption per person in Oceania surpassed that in Europe, and the Americas consumed 41 times more than Asia and 103 times more than Africa. Nationally, consumption per head of population in Germany and Iceland exceeded that in North America. Analgesics were the most commonly consumed controlled opioids, with oxycodone, morphine and methadone leading the way.

Compared with previous research, 8 there has been a shift in consumption from North American countries to Germany and Iceland. This shift may be due to mitigation strategies (e.g. prescription monitoring programmes and pill mill laws) in North America which are reducing medical access to opioids. ${ }^{38-40}$ However, this restriction has had devastating consequences. Opioid-dependent people without access to opioid substitution therapy, 


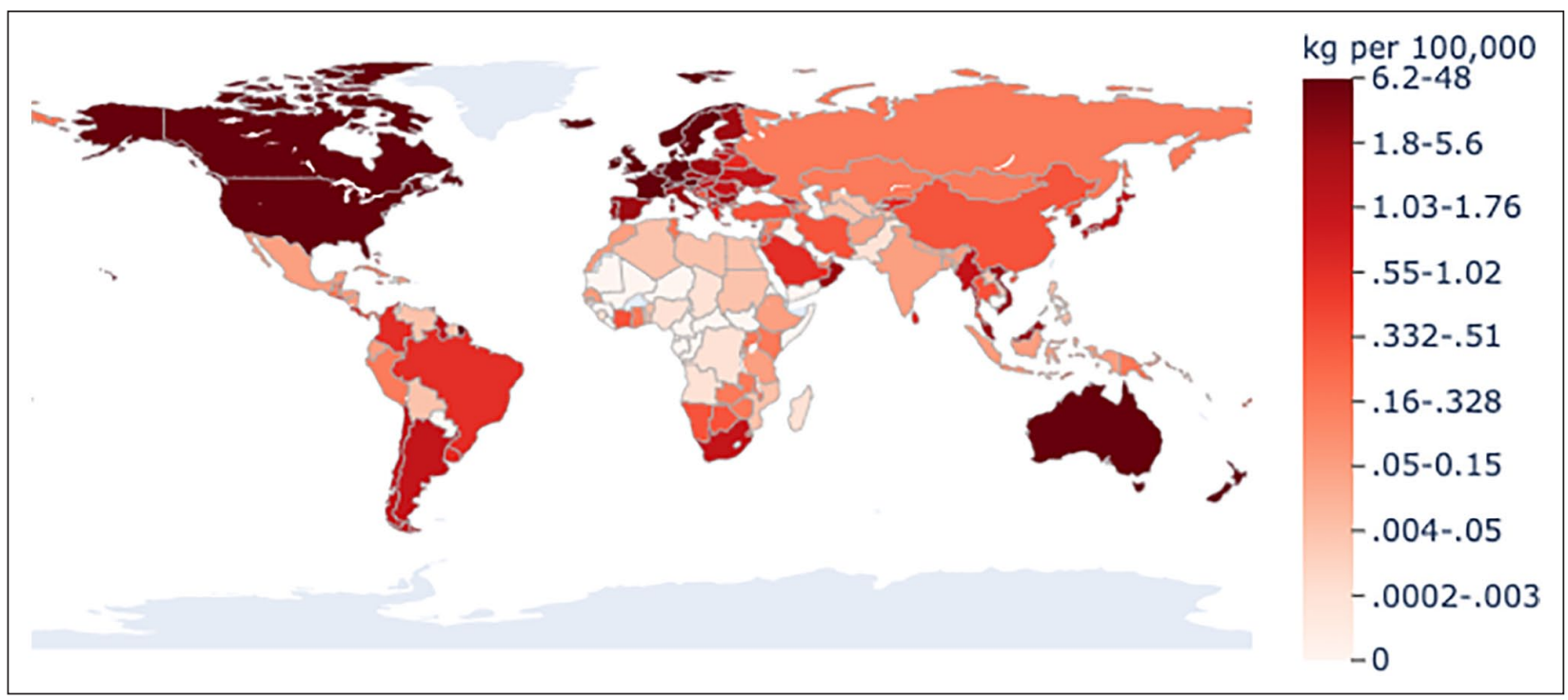

Figure 2. Annual mean consumption of controlled opioids for 2015-2017 grouped by deciles for all countries and nonmetropolitan territories ( $n=214$ ). Consumption refers to the total amount of controlled opioids distributed for medical purposes and excludes recreational use; it was calculated by determining the 3-year mean for 2015-2017 and dividing this by the 2016 population for each country. Data were obtained from the International Narcotics Control Board.

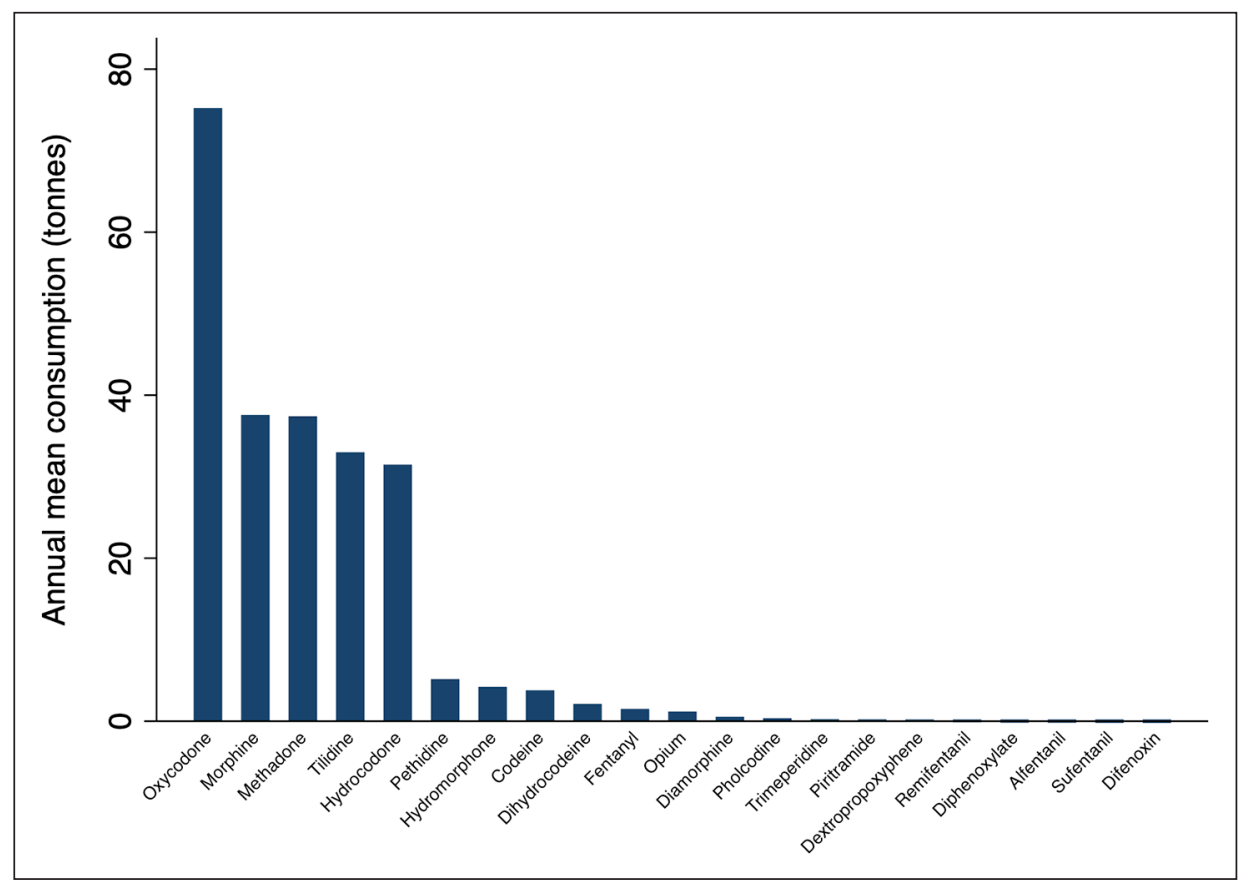

Figure 3. Annual mean consumption (tonnes) in 2015-2017 by type of opioid where consumption refers to the total amount of controlled opioids distributed for medical purposes and excludes recreational use in all countries and nonmetropolitan territories ( $\mathrm{n}=214$ ) with data provided by the International Narcotics Control Board.

such as methadone, have moved to illicit sources of opioids, and unintended overdose deaths have rapidly increased. ${ }^{41}$ Lessons from the North American opioid crisis must be considered by all countries when designing strategies and clinical guidelines that improve access to opioids while limiting dependence and harm. There are currently no National Institute for Health and Care Excellence (NICE) guidelines for prescribing opioids in the United Kingdom, and the WHO has recently retracted its two main guidelines for opioid use because of interference by opioid manufacturers. ${ }^{42,43}$ Thus, a coordinated effort is needed across all countries to 
develop evidence-based guidelines that promote the appropriate use of opioids and warn against the significant harms of opioids, and support non-pharmacological approaches to managing chronic pain.

We found disparities and wide variations in consumption regionally and nationally. Previous research has highlighted many barriers to accessing opioids, including the absence of training, fear of dependence and diversion, problems in sourcing, the complexities of regulating opioids as internationally controlled substances, and cultural and social attitudes. ${ }^{8,23-25}$ In countries with low opioid consumption, there may be access to non-opioid alternatives, such as paracetamol for pain and ketamine or bupivacaine for anaesthesia, and varying national guidance that may impact the use of controlled opioids. The WHO and the INCB have made efforts to encourage governments to have balanced policies and health systems that ensure opioids are both available and accessible to those in need. However, our findings question whether much has been achieved to overcome the barriers, as disparities in access to opioids persist.

While access to opioids for acute pain, palliative care and cancer pain should continue to be advocated, there is growing concern that pharmaceutical companies have started US-style marketing techniques in low- and middle-income countries. ${ }^{44,45}$ Prescribers in these countries must therefore be prepared to deal with marketing campaigns to prescribe and use opioids and be educated about the consequences of conflicts of interests until sanctions can be enforced to prevent a North American opioid crisis.

\section{Strengths and limitations}

We included data from all countries and non-metropolitan territories for all available types of opioids (anaesthetics, analgesics, antidiarrheals, cough suppressants and opioid substitution therapies). Thus, our findings are representative of the global population between 2015 and 2017. We standardized consumption using 2016 population statistics to create a rate per person, which assumes that opioids were consumed by individuals of all ages, including children. However, it is not possible to elucidate what proportion of people consumed or received opioids in each country. Data for opioids that are not regulated as internationally controlled substances (e.g. tramadol and buprenorphine) are not reported to the INCB. Thus, this analysis represents the consumption of internationally controlled opioids. However, we have included 225 substances, which incorporate all types of available opioids rather than focusing on analgesics or a subset of opioids, as previous researchers have done. . $^{910,21,32}$ We found 35 countries that reported no consumption of opioids to the INCB, and it is unclear whether this reflects actual consumption, as data may be late, unreported or submitted inaccurately, as previously described. ${ }^{8,10}$ We categorized opioids using the ATC index; however, there are opioids, such as fentanyl, that may be used as analgesics and anaesthetics, which were not accounted for.

The effects of opioids vary by type and weight (e.g. $10 \mathrm{mg}$ of morphine will have a greater effect than 10 mg of codeine), which morphine equivalent conversion would account for if accurate conversion were possible. We measured consumption using weight in mg adjusted for country population, as potency ratios and defined daily dose (DDD) conversion factors are not available for all opioid substances included in our analysis. The WHO created DDDs, a technical measure given to most medicines with an ATC code, defined as 'the assumed average maintenance dose per day for a drug used for its main indication in adults'. ${ }^{46} \mathrm{~A}$ DDD provides an estimate of consumption rather than the actual use of a drug, and its use in opioid research is discouraged. ${ }^{47}$ Thus, our findings may not be easily comparable with those of most previous studies, ${ }^{7,8,31}$ in which consumption has been measured using oral morphine equivalents or DDDs.

\section{Conclusion}

The consumption of controlled opioids remains low in most of the world while being extremely high in a few countries. Countries with very high rates of consumption may need to implement measures that promote appropriate prescribing. Governments and international bodies should work together to update and develop clinical guidelines, policies, and health systems that promote access to opioids for acute pain, cancer pain, palliative care, anaesthesia and opioid dependence, assess the evidence base of opioids as antidiarrheals and cough suppressants, implement strategies to reduce the harms of opioids, and mitigate the outcomes that arise when pharmaceutical companies act only in their own interests and not those of patients.

\section{Acknowledgements}

The authors thank the International Narcotic Control Board for sharing the raw narcotic consumption data.

\section{Conflict of interest}

The author(s) declared the following potential conflicts of interest with respect to the research, authorship and/or publication of this article: G.C.R. was financially supported by the National Institute for Health Research (NIHR) School for Primary Care Research (SPCR), the Naji Foundation and the Rotary Foundation to study for a Doctor of Philosophy (2017-2020) but no longer has any financial COIs. G.C.R. is 
an Associate Editor of BMJ Evidence-Based Medicine. J.K.A. has published articles and edited textbooks on adverse drug reactions and interactions and has often given medicolegal advice, including appearances as an expert witness in coroners' courts, often dealing with the adverse effects of opioids. K.R.M. has received funding to conduct research from the NIHR and the Royal College of General Practitioners (RCGP) to conduct independent research. C.H. is an NIHR Senior Investigator and has received expenses and fees for his media work, received expenses from the WHO and FDA, and holds grant funding from the NIHR SPCR and the NIHR SPCR Evidence Synthesis Working Group (Project 380), the NIHR BRC Oxford and the WHO. On occasion, C.H. receives expenses for teaching EBM and is also paid for his GP work in National Health Service (NHS) out of hours (contract with the Oxford Health NHS Foundation Trust). C.H. is the Director of the CEBM. The views expressed are those of the authors and not necessarily those of the NHS, the NIHR or the Department of Health and Social Care.

\section{Contributorship}

G.C.R. designed the study, obtained the data, analysed the data and wrote the manuscript. J.K.A., K.R.M. and C.H. advised on study design, provided supervisory support and provided advice, edits and comments on the manuscript.

\section{Funding}

The author(s) received no financial support for the research, authorship and/or publication of this article.

\section{Guarantor}

G.C.R. is the guarantor of this study.

\section{ORCID iD}

Georgia C Richards (iD https://orcid.org/0000-0003-0244 $-5620$

\section{Supplemental material}

Supplemental material for this article is available online.

\section{References}

1. Lohman D, Schleifer R and Amon JJ. Access to pain treatment as a human right. BMC Med 2010; 8(1): 8, http://bmc medicine.biomedcentral.com/articles/10.1186/1741 -7015-8-8 (accessed 29 March 2020).

2. WHO. World Health Organization model list of essential medicines, 21st list. Geneva: World Health Organization, 2019, https://creativecommons.org/licenses/by-ncsa/3.0/igo/ (accessed 25 October 2020).

3. Richards GC, Aronson JK, Heneghan C, et al. Relation between opioid consumption and inclusion of opioids in 137 national essential medicines lists. BMF Glob Health 2020; 5(11): e003563.

4. Ventafridda V, Saita L, Ripamonti C, et al. WHO guidelines for the use of analgesics in cancer pain. Int $\mathcal{F}$ Tissue React 1985; 7(1): 93-96, https://pubmed.ncbi.nlm.nih. gov/2409039/ (accessed 2 March 2021).
5. Ballantyne JC, Kalso E and Stannard C. WHO analgesic ladder: a good concept gone astray. BMF 2016; 352: i20, http://www.bmj.com/ (accessed 4 March 2021).

6. Levy N, Sturgess J and Mills P. 'Pain as the fifth vital sign' and dependence on the 'numerical pain scale' is being abandoned in the US: why? Br $\mathcal{F}$ Anaesth 2018; 120(3): 435-438.

7. Scholten WK, Christensen AE, Olesen AE, et al. Quantifying the adequacy of opioid analgesic consumption globally: an updated method and early findings. $A m \mathcal{F}$ Public Health 2019; 109(1): 52-57.

8. Berterame S, Erthal J, Thomas J, et al. Use of and barriers to access to opioid analgesics: a worldwide, regional, and national study. Lancet 2016; 387(10028): 1644-1656.

9. Hastie BA, Gilson AM, Maurer MA, et al. An examination of global and regional opioid consumption trends 1980-2011. I Pain Palliat Care Pharmacother 2014; 28(3): 259-275.

10. Gilson AM, Maurer MA, Ryan KM, et al. Using a morphine equivalence metric to quantify opioid consumption: examining the capacity to provide effective treatment of debilitating pain at the global, regional, and country levels. F Pain Symptom Manage 2013; 45(4): 681-700.

11. United Nations Single Convention on Narcotic Drugs, 1961, https:/www.emcdda.europa.eu/drugs-library/single-convention-narcotic-drugs-1961_en (1961, accessed 6 August 2020).

12. INCB. Mandate-functions. Vienna: International Narcotic Control Board, 2019, https://www.incb.org/incb/en/ about/mandate-functions.html (accessed 29 April 2020).

13. INCB. Narcotic drugs - estimated world requirements for 2020 - statistics for 2019. Vienna: International Narcotic Control Board, 2019, https:/www.incb.org/documents/ Narcotic-Drugs/Technical-Publications/2020/Narcotic Drugs_Technical_publication_2020.pdf (accessed 25 October 2020).

14. Curtis HJ, Croker R, Walker AJ, et al. Opioid prescribing trends and geographical variation in England, 19982018: a retrospective database study. Lancet Psychiatry 2019; 6(2): 140-150.

15. Davies E, Sewell B, Jones M, et al. Examining opioid prescribing trends for non-cancer pain using an estimated oral morphine equivalence measure: a retrospective cohort study between 2005 and 2015. BfGP Open 2020; 5: bjgpopen20X101122, https://bjgpopen. org/content/early/2020/11/30/bjgpopen $20 X 101122$ (accessed 7 January 2021).

16. Kalkman GA, Kramers C, Van Dongen RT, et al. Trends in use and misuse of opioids in the Netherlands: a retrospective, multi-source database study. Lancet Public Health 2019; 4: e498-e505, www.thelancet.com/ (accessed 29 October 2019).

17. Chenaf C, Kaboré JL, Delorme J, et al. Prescription opioid analgesic use in France: trends and impact on morbidity-mortality. Eur F Pain 2019; 23(1): 124-134.

18. Gomes T, Khuu W, Martins D, et al. Contributions of prescribed and non-prescribed opioids to opioid related deaths: population based cohort study in Ontario, Canada. BMF 2018; 362: k3207, https://www.bmj.com/content/362/bmj.k3207 
19. Richards GC, Mahtani KR, Muthee TB, et al. Factors associated with the prescribing of high-dose opioids in primary care: a systematic review and meta-analysis. BMC Med 2020; 18(1): 68, https:// bmcmedicine.biomedcentral.com/articles/10.1186/ s12916-020-01528-7 (accessed 31 March 2020).

20. Karanges EA, Buckley NA, Brett J, et al. Trends in opioid utilisation in Australia, 2006-2015: insights from multiple metrics. Pharmacoepidemiol Drug Saf 2018; 27(5): 504-512.

21. Duthey B and Scholten W. Adequacy of opioid analgesic consumption at country, global, and regional levels in 2010, its relationship with development level, and changes compared with 2006. F Pain Symptom Manage 2014; 47(2): 283-297.

22. Seya MJ, Gelders Achara OU, Milani B, et al. A first comparison between the consumption of and the need for opioid analgesics at country, regional, and global levels. F Pain Palliat Care Pharmacother 2011; 25(1): 6-18.

23. Bosetti C, Santucci C, Radrezza S, et al. Trends in the consumption of opioids for the treatment of severe pain in Europe, 1990-2016. Eur F Pain 2018; 23(4): 697707, https://onlinelibrary.wiley.com/doi/abs/10.1002/ ejp.1337 (accessed 10 January 2020).

24. Vranken MJM, Mantel-Teeuwisse AK, Schutjens Scholten WK, et al. Access to strong opioid analgesics in the context of legal and regulatory barriers in eleven central and Eastern European countries. F Palliat Med 2018; 21(7): 963-969.

25. Vranken MJM, Lisman JA, Mantel-Teeuwisse AK, et al. Barriers to access to opioid medicines: a review of national legislation and regulations of 11 central and eastern European countries. Lancet Oncol 2016; 17(1): e13-e22.

26. Larjow E, Papavasiliou E, Payne S, et al. A systematic content analysis of policy barriers impeding access to opioid medication in central and Eastern Europe: results of ATOME. F Pain Symptom Manage 2016; 51: 99-107.

27. Linge-Dahl L, Vranken M, Juenger S, et al. Identification of challenges to the availability and accessibility of opioids in twelve European countries: conclusions from two ATOME six-country workshops. F Palliat Med 2015; 18(12): 1033-1039.

28. Cherny NI, Baselga J, de Conno F, et al. Formulary availability and regulatory barriers to accessibility of opioids for cancer pain in Europe: a report from the ESMO/EAPC Opioid Policy Initiative. Ann Oncol 2010; 21: 615-626.

29. Cherny NI, Cleary J, Scholten W, et al. The Global Opioid Policy Initiative (GOPI) project to evaluate the availability and accessibility of opioids for the management of cancer pain in Africa, Asia, Latin America and the Caribbean, and the Middle East: introduction and methodology. Ann Oncol 2013; 24(Suppl. 11): xi7-xi13, https://linkinghub. elsevier.com/retrieve/pii/S0923753419374526

30. Degenhardt L, Grebely J, Stone J, et al. Global patterns of opioid use and dependence: harms to populations, interventions, and future action. Lancet 2019; 394: 1560-1579.

31. Knaul FM, Farmer PE, Krakauer EL, et al. Alleviating the access abyss in palliative care and pain relief - an imperative of universal health coverage: the Lancet Commission report. Lancet 2018; 391: 1391-1454.

32. Gilson AM, Maurer MA, Lebaron VT, et al. Multivariate analysis of countries' government and health-care system influences on opioid availability for cancer pain relief and palliative care: more than a function of human development. Palliat Med 2013; 27(2): 105-114.

33. Thomas J. Availability of narcotic drugs for medical use. Vienna: International Narcotic Control Board, 2019, https:/www.incb.org/incb/en/narcotic-drugs/Availability/availability.html (accessed 19 November 2019).

34. StataCorp. Create variable containing percentiles. Stata documentation, https://www.stata.com/manuals/dpctile. pdf (2020, accessed 16 March 2021).

35. StataCorp. Stata Statistical Software, Release 16. College Station, TX: StataCorp, 2019.

36. Richards GC, Aronson JK, Heneghan C, et al. Global, regional \& national consumption of controlled opioids. OSF, https://osf.io/6nzds/ (2020, accessed 8 February 2021).

37. Richards G. opioid_emls_maps/Opioid consumption maps.ipynb. Github. p.georgiarichards/opioid_emls_ maps, https:/github.com/georgiarichards/opioid_emls_ maps/blob/master/Opioidconsumptionmaps.ipynb (2020, accessed 8 February 2021).

38. Schieber LZ, Guy GP, Seth P, et al. Trends and patterns of geographic variation in opioid prescribing practices by state, United States, 2006-2017. FAMA Netw Open 2019; 2(3): e190665.

39. Rutkow L, Chang HY, Daubresse M, et al. Effect of Florida's Prescription Drug Monitoring Program and pill mill laws on opioid prescribing and use. $\mathcal{F} A M A$ Intern Med 2015; 175(10): 1642-1649.

40. Geissert P, Hallvik S, Van Otterloo J, et al. High-risk prescribing and opioid overdose: prospects for prescription drug monitoring program-based proactive alerts. Pain 2018; 159(1): 150-156, http://journals.lww.com/00006396201801000-00019 (accessed 11 May 2020).

41. Ciccarone D. Fentanyl in the US heroin supply: a rapidly changing risk environment. Int $\mathcal{F}$ Drug Policy 2017; 46: 107-111.

42. Dyer O. WHO drops opioid guidelines after criticism of corporate influence. BMF 2019; 365: 14374.

43. NICE. Project information| Chronic pain: assessment and management, https://www.nice.org.uk/guidance/indevelopment/gid-ng10069 (2021, accessed 5 March 2021).

44. Kinetz E. Fake doctors, pilfered medical records drive Oxy China sales. Associated Press, 20 November, https:// apnews.com/4122af46fdba42119ae3db30aa13537c (2019, accessed 14 May 2020).

45. Palinkas LA. Opioid use epidemic in Mexico: global solutions to a global problem. Am F Public Health 2019; 109(1): 26-27.

46. WHO. Defined daily dose (DDD), https://www.who. int/tools/atc-ddd-toolkit/about-ddd (2021, accessed 5 March 2021).

47. Nielsen S, Gisev N, Bruno R, et al. Defined daily doses (DDD) do not accurately reflect opioid doses used in contemporary chronic pain treatment. Pharmacoepidemiol Drug Saf 2017; 26(5): 587-591. 